\title{
The faint end luminosity function of compact galaxy groups
}

\author{
E. Krusch ${ }^{1}$, D. Rosenbaum ${ }^{1}$, R.-J. Dettmar ${ }^{1}$, D. J. Bomans ${ }^{1}$, C. L. Taylor ${ }^{2}$, G. Aronica ${ }^{1}$, and T. Elwert ${ }^{3}$ \\ 1 Astronomisches Institut, Ruhr-Universität Bochum, Universitätsstr. 150, 44780 Bochum, Germany \\ e-mail: krusch@astro.ruhr-uni-bochum.de \\ 2 Department of Physics and Astronomy, California State University Sacramento, 6000 J Street, Sacramento, CA 95819, USA \\ 3 Department of Physics and Astronomy, University of Kentucky, Lexington, KY 40506, USA
}

Received 14 November 2005 / Accepted 28 August 2006

ABSTRACT

\begin{abstract}
We present the combined Luminosity Function of five nearby Hickson Compact Groups. We identify at the faint end the dwarf galaxy group members using the red sequence of the Color Magnitude Diagram from our deep $2.2 \mathrm{~m}$ WFI photometry of Hickson Compact Groups 16, 19, 30, 31 and 42. We find that the Luminosity Function of compact groups shows a bimodal distribution of bright galaxies and a dwarf galaxy population as it is known for galaxy clusters, but not for field galaxies. From measuring the field to field variance of background galaxies fulfilling the red sequence criterion coincidentally, a lower limit for the significance of the dwarf galaxy peak in the Luminosity Function was derived. With this method we determined the dwarf galaxy peak to have a significance of more than $2.6 \sigma$. Thus, compact groups can be classified as bound systems like galaxy clusters, which also accommodate two galaxy populations. Hence, it seems less unlikely that compact groups are chance configurations of individual galaxies. Our result shows that compact groups are building blocks of galaxy clusters underlining a hierarchical model of structure formation and galaxy evolution. Further we note that the existence of a significant number of satellites in bound systems like compact groups is predicted by $\Lambda$ Cold Dark Matter theory.
\end{abstract}

Key words. galaxis: dwarf - galaxies: clusters: general - galaxies: photometry

\section{Introduction}

Theoretical models of structure formation predict the existence of many low-mass halos in bound systems (Benson et al. 2003; Gottlöber et al. 2001; Klypin et al. 1999; Moore et al. 1999). If these halos are birthplaces of dwarf galaxies, there should be many more dwarf galaxies than giant galaxies in bound systems. In this publication we will discuss the Luminosity Function (LF) of Compact Galaxy Groups (CGs), in which we find indeed a high number of dwarf galaxy group members.

Using the LF it is possible to compare quantitatively the galaxy content in different environments. Comparing the LFs of field galaxies to that of a bound system like galaxy clusters, a difference in the shape of the LF can be found. Investigations of the LFs of field galaxies derive a weakly rising slope at the faint end (e.g. Blanton et al. 2001). In contrast the LF of galaxy clusters shows a rising LF at the faint end (e.g. Trentham \& Tully 2002; Sandage et al. 1985) indicating the existence of an additional dwarf galaxy population.

Several investigations on LFs of CGs show controversial findings. Former results of LFs of CGs exhibit similarities to LFs of the field (Ribeiro et al. 1994; Kodaira et al. 1991; Zepf \& Whitmore 1991; Mendes de Oliveira \& Hickson 1994; Hickson 1982), whereas recent results point to the existence of a distinct dwarf galaxy population in CGs (Miles et al. 2004; Zabludoff \& Mulchaey 2000; Hunsberger et al. 1998). The differing LFs may be explained by the fact that the investigation of galaxy systems generally proves to be difficult due to observational selection effects in samples and uncertainties concerning the group membership of galaxies. The LF of HCGs especially at the faint end can give important clues to the understanding of structure formation.

\section{Data and data reduction}

We compiled a sample of five HCGs (HCG 16, 19, 30, 31 and 42) using the ESO/La Silla $2.2 \mathrm{~m}$ telescope in combination with the Wide Field Imager (WFI). The WFI acquires a large field of view $0.54^{\circ} \times 0.57^{\circ}$. The data were obtained in Johnson-Cousins $B$ and $R$ broad band filters (B/99, Rc/162). Exposure times for the $B$ band images amount to $5400 \mathrm{~s}$ and to $2700 \mathrm{~s}$ in the $R$ band. By means of that a surface brightness limit of $27 \mathrm{mag} / \mathrm{arcsec}^{2}$ in the $B$ band and $26 \mathrm{mag} / \operatorname{arcsec}^{2}$ in the $R$ band were reached.

The data were reduced using IRAF (Image Reduction and Analysis Facility), and MSCRED version 2.0 (Valdes 1998). A more detailed discussion of the data reduction will be given in Krusch et al. The reduced mosaic images were scanned for dwarf galaxies using the program Source Extractor (Bertin \& Arnouts 1996). After sky subtraction all objects with a threshold $\geq 1.1 \sigma$ in $B$ and $R$ above the sky level were extracted. Finally, all galaxies with a photometric error larger than $0.1 \mathrm{mag}$ and a physical size smaller than the smallest identified dwarf galaxy of the Local Group, LGS 3 (Mateo 1998) projected to the distance of the $\mathrm{CG}$ were excluded.

Determination of group membership is a common problem when studying the faint galaxy population in clusters and groups. The most reliable method of taking spectra to determine redshifts for all candidates is a very telescope-time consuming task and is also mostly limited to the brighter galaxies (Zabludoff \& Mulchaey 1998). Therefore, we developed a new method to determine the group membership of the galaxies from a preselection using Color Magnitude Diagrams (CMDs). Investigations of galaxy clusters show that $\mathrm{dE}$ galaxies dominate a certain area in the CMD, which was determined for the Coma and Perseus Cluster (Secker \& Harris 1997; Conselice et al. 2002). This 


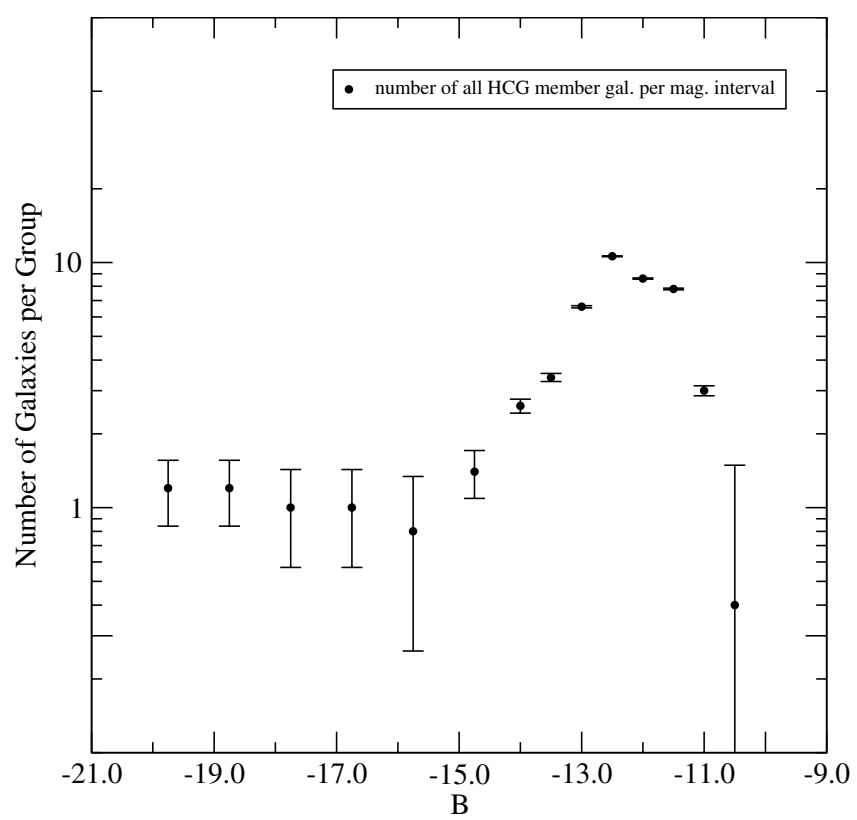

Fig. 1. $B$ band Luminosity Function of 5 HCGs per group and per magnitude interval. Galaxy members lying in the interval $-20.25 \mathrm{mag} \leq$ $B \leq-16.25 \mathrm{mag}$ were binned in $1 \mathrm{mag}$ steps and reflect the number of giant galaxies per interval per group. They are subject to small number statistics, which is expected due to the definition of HCGs (see Hickson 1982). Faint galaxies, lying in the interval $-16.25 \mathrm{mag} \leq B \leq$ $-10.25 \mathrm{mag}$, were binned in $0.5 \mathrm{mag}$ steps.

relation was used for the determination of the high probability group membership of dwarf elliptical galaxies in the CMDs of the sample HCGs. All galaxies in the red sequence are high probability $\mathrm{dE}$ group members and all galaxies bluer than the red sequence are high probability dwarf irregular galaxy candidates. All these galaxies were inspected concerning their morphology, isophotes, and radial light profile.

In order to measure the quality of this method and to estimate the significance of the dwarf galaxy peak in the LF (Fig. 1) the field to field variance of background galaxies getting into the red sequence criterion coincidentally was determined the following way. First, for each of the examined HCGs the radius containing $90 \%$ of the high probability $\mathrm{dE}$ group members was determined. These galaxies were assumed to be HCG members forming the signal of the red sequence method. All dwarf galaxies beyond this radius, but fulfilling the red sequence criterion, located at the edges of the WFI images, were assumed to be non-members of the HCGs. These galaxies represent the noise in the red sequence. Then, the field to field variance was determined by number counting these non-member galaxies within four apertures of same size placed at the edge of the WFI field of view. Then the signal was normalized with the ratio of area covered by the $90 \%$ radius and the aperture within the field to field variance was measured. This analysis was performed for all five HCGs, resulting in a total signal to noise of $2.6 \sigma$. Expecting the assumption that all red sequence dwarf galaxies beyond the $90 \%$ radius are non-members to be not entirely accurate, the determined significance is only a lower limit to the true signal to noise ratio.

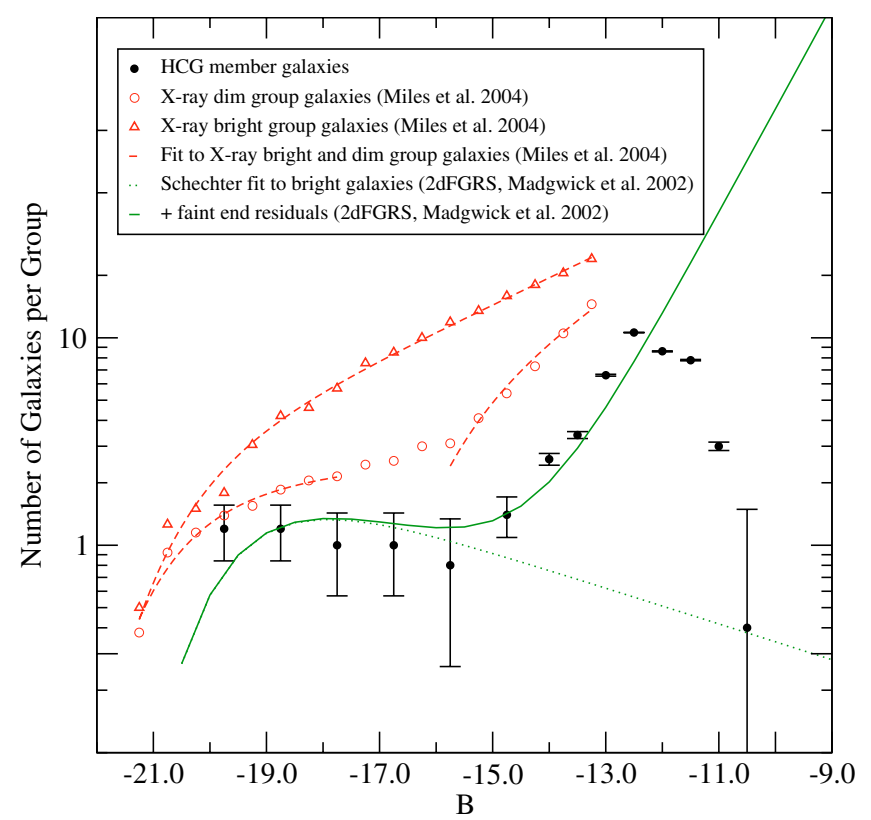

Fig. 2. The same as Fig. 1 plus data for the LF of X-ray dim (empty circles) and X-ray bright (triangles) galaxy groups and the according Schechter fits (dashed lines) investigated by Miles et al. (2004). The dotted line represents the Schechter function for bright field galaxies of passive star forming galaxies of the 2dFGRS and the solid line represents the combination of this with the analytic form for the faint-end residuals (Madgwick et al. 2002).

\section{The faint end luminosity function of Hickson compact groups}

With our deep CCD-mosaic observations we reach a much lower luminosity limit than previous LF studies of HCGs which is the main improvement compared to LFs of HCGs published in literature. The LF of the HCGs (Fig. 1) was determined for member galaxies of the sample HCGs down to a magnitude of $B=-12.5$ mag. The distribution of all sample HCG member galaxies can be divided into two qualitatively distinct parts. At the bright end, from $\sim-20$ to $-16 \mathrm{mag}$, the distribution can be described by a plateau, which is slightly declining towards fainter magnitude intervals. Beginning at the magnitude interval of $\sim-15$ mag the number of galaxies per interval unit rises steeply reaching a maximum at $\sim-12 \mathrm{mag}$. The steep drop at right is due to a rising incompleteness of the data reaching the detection limit. The LF of HCGs clearly indicates the existence of two galaxy populations: bright galaxies forming the plateau at left in the LF and dwarf galaxies representing the rise at right.

\subsection{Comparison of the LF of HCGs and of other environments}

The two quantitatively distinct parts (Fig. 1), representing the bright and dim galaxies were also found by Miles et al. (2004) (see Fig. 2). They divided their sample into X-ray $\operatorname{dim}\left(L_{\mathrm{X}} \leq\right.$ $\left.10^{41.7} \mathrm{erg} / \mathrm{s}\right)$ or low velocity dispersion and X-ray bright $\left(L_{\mathrm{x}} \geq\right.$ $10^{41.7} \mathrm{erg} / \mathrm{s}$ ) groups. The latter (triangles) do not show such a division, although the LF of the X-ray dim groups (to which all HCGs of their sample belong; empty circles) does. The dashed lines represent the Schechter (1976) fits to the bright and faint part of X-ray dim galaxies and to the X-ray bright galaxies. The similarity between the LFs of X-ray dim groups and HCGs 
implies that HCGs may be X-ray dim systems with a significant distinction between the two different galaxy populations.

Flint et al. (2001) investigated the Leo I Group at $\sim 10 \mathrm{Mpc}$ in order to study the galaxy LF at $z \approx 0$. Although this group is not a compact galaxy group, the distribution of galaxies as a function of magnitude corresponds to that of our HCG sample. Leo I and our HCG sample have nearly the same faint end slope ( $B \geq-15 \mathrm{mag}$ ) implying that these groups consist of almost the same population and thus the $\mathrm{dE}$ galaxies may have the same formation history. Therefore it seems that the dEs in the HCGs have not yet participated to a significant degree in the dynamical evolution of HCGs.

Madgwick et al. (2002) examined the LF of the field investigating the 2dFGRS ( 2 degree Field Galaxy Redshift Survey) galaxies by dividing them into four spectral types using principal component analysis and calculating the LF for each of them. They showed that the faint-end slope systematically steepens while moving from passive to active star-forming galaxies. They also found that a single Schechter function provides a poor fit to the very faint galaxies of the passive star forming galaxies suggesting the existence of a dwarf elliptical galaxy population. Thus, they fitted a Schechter function to the bright galaxies (Fig. 2, dotted line) and a combination of this with their analytic form for the faint-end residuals (Fig. 2, solid line). Their fits stop at $M_{B}=-13 \mathrm{mag}$. In order to compare the faint end of the LF of HCGs to this fit we extrapolated it to $M_{B}=-9 \mathrm{mag}$. The rise in the LF function of passive star-forming galaxies (Fig. 2, solid line) coincides with the slope at the faint end of the LF of HCGs $\left(M_{B} \geq-15 \mathrm{mag}\right)$. This can be explained by the fact that galaxy groups had not been excluded from the $2 \mathrm{dF}$ sample and dominate the faint end.

Therefore one of the main analogous feature of LFs of HCGs, X-ray dim groups and the Leo I group is a deficit in intermediate magnitude galaxies.

\subsection{Comparison between the LF of HCGs and the predictions of the CDM theory}

Theories of structure formation and $\Lambda \mathrm{CDM}$ theory predict a large number of dwarf halos resulting in a steep rise $(\alpha \leq-1.6)$ at the faint end of the LF (e.g. Gottlöber et al. 2001; Klypin et al. 1999). A Schechter fit to the faint end of HCG galaxies of our sample (Fig. 3, solid line) results in $\alpha=-1.73$. The dotted and dashed lines represent the same Schechter LF but with $\alpha=-1.8$ and $\alpha=-1.6$ as predicted by theory. They suggest that dark halos in dense environments formed early in the history of the Universe, but that dark halos in diffuse and non-evolved environments assembled much later. Hence, the dark halos in evolved environments, such as the Virgo Cluster, could collect gas that could later be processed into stars, but dark halos in nonevolved environments could not. As a result more dwarf galaxies per unit total mass could be found in evolved environments than in non-evolved environments. Further, Trentham \& Tully (2002) determined the galaxy LF in five different local environments. They found that in all cases fewer dwarfs were detected than the numbers of low-mass halos anticipated by theory. However, the steep rise of the LF of HCG member galaxies (Fig. 3) now established, may be compatible with the values predicted by $\Lambda \mathrm{CDM}$ and structure formation models.

\section{Discussion and conclusions}

Our investigation of the LF of HCGs shows a significant break dividing the bright and faint galaxy population. The break in

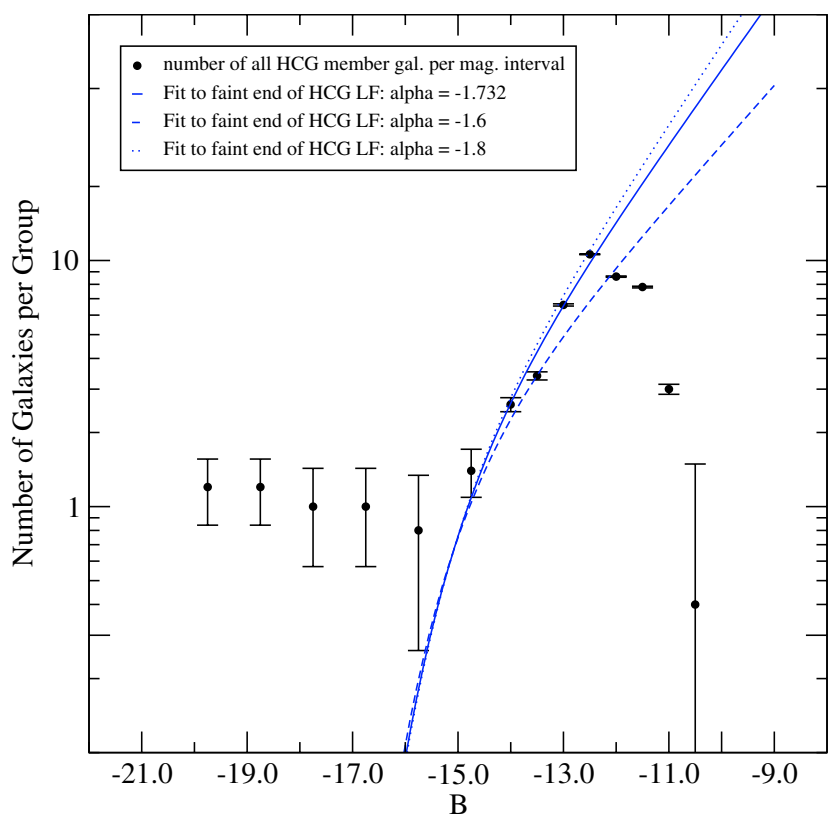

Fig. 3. The same as Fig. 1. The solid line represents the Schechter fit to the faint end of the LF. The dotted and dashed lines represent this LF with $\alpha$ values predicted by $\Lambda \mathrm{CDM}$ theory.

the LF of HCGs at $B \approx-16$ mag cannot be due to background galaxies at $B \approx-22 \mathrm{mag}$ (e.g. Koo \& Kron 1992) for two reasons. First, our selection depends on the red galaxies for which the change in slope is much less pronounced and second, the break of the background galaxies at the distance of the HCGs would be in the range from -10.5 to $-11.5 \mathrm{mag}$. Independently this break was also found in the LF of galaxy systems, e.g. X-ray dim groups (Miles et al. 2004, Fig. 2 open circles) and the Leo I group (Flint et al. 2001). Miles et al. (2004) argued that the lower the velocity dispersion of the groups, the higher the evidence for the formation of few bright galaxies at the bright end of the LF. Since a power law relation exists between the luminosity and the velocity dispersion (Helsdon \& Ponman 2000), they assumed that X-ray dim groups exhibit a lower velocity dispersion than X-ray bright groups.

Miles et al. (2004) used simulations which showed that in the low velocity dispersion environment dynamical friction is expected to be more effective in ensuring that galaxies fall towards the centre of the group potential, as well as facilitating galaxy interactions and mergers and thus deplete the intermediate luminosity galaxies leading to the formation of a few giant central galaxies at the bright end. This would result in a drop at the intermediate luminosity galaxies, while the faint end of the LF shows no appreciable change in slope. We further argue that with an ongoing evolution of the galaxy group dwarf galaxies fall into the group potential and merge with high-mass galaxies. In this case the level of the faint end of the total group LF can be expected to diminish.

The lack of X-ray emission in some of the X-ray dim systems could mean that they are yet to form virialised entities. In that case they are late-forming systems, which are only now reaching a state of high density. The most likely interpretation of the shape of the LF thus seems to be that in X-ray dim systems, HCGs and the Leo I group, most of the galaxy mergers responsible for the depression in the LF have taken place in the recent past. In contrast X-ray bright high velocity systems are probably not undergoing much merging at all right now (due to 
high galaxy velocities). It seems that the mergers, which have affected their morphological mix and the LFs, may have taken place at earlier epochs, quite probably in a variety of structures, which have merged to form the group we see now. In the field, where mergers are rare, the intermediate-luminosity break in the LF is not expected to appear, although the LF of passive star forming galaxies, shows two components: a bright and a faint galaxy population (e.g. Madgwick et al. 2002, Fig. 2).

That rich clusters of galaxies in general do not have bimodal LFs of this kind can be seen from the composite cluster LFs found by de Propris et al. (2003) from the $2 \mathrm{dF}$ galaxy redshift survey. Clearly if one were to create a cluster by merging a set of X-ray dim groups, the result would be a cluster with a break in the LF as weakly indicated in Coma (Mobasher et al. 2003) and Virgo (Trentham \& Hodgkin 2002). However, their break in the LF is less pronounced as that in our HCG-LF.

It therefore seems that the diversity in the LFs of richer clusters reflects a diversity in their formation history. White et al. (1993) showed Coma to be a rather common cluster, which shows evidence for a turbulent recent history involving multiple subcluster mergers. They speculated that clusters with strong LF breaks may have been assembled primarily by multiple mergers of low velocity dispersion groups. The results presented here suggest that compact galaxy groups and X-ray dim groups are sites of recent dynamical evolution. Their X-ray bright counterparts and galaxy clusters may have gone through this stage in the distant past with the LF evolving such that its faint end gradually depleted.

Acknowledgements. Many thanks to Volker Müller for constructive collaboration and many discussions especially concerning the theoretical part and to Gottfried Beyvers for proof reading and useful suggestions. This work was supported by the German Deutsche Forschungsgemeinschaft, DFG project number GRK 787. The observations were collected at the European Southern Observatory, Chile (Observing Programme 64.N-0007A) and we made use of the NASA/IPAC Extragalactic Database (NED) which is operated by the Jet Propulsion Laboratory, California Institute of Technology, under contract with the National Aeronautics and Space Administration.

\section{References}

Benson, A. J., Bower, R. G., Frenk, C. S., et al. 2003, ApJ, 599, 38 Bertin, E., \& Arnouts, S. 1996, A\&AS, 117, 393

Blanton, M. R., Dalcanton, J., Eisenstein, D., et al. 2001, AJ, 121, 2358 Conselice, C. J., Gallagher, J. S., \& Wyse, R. F. G. 2002, AJ, 123, 2246 de Propris, R., Colless, M., Driver, S. P., et al. 2003, MNRAS, 342, 725 Flint, K., Metevier, A. J., Bolte, M., \& Mendes de Oliveira, C. 2001, ApJS, 134, 53

Gottlöber, S., Klypin, A., \& Kravtsov, A. V. 2001, ApJ, 546, 223

Helsdon, S. F., \& Ponman, T. J. 2000, MNRAS, 319, 933

Hickson, P. 1982, ApJ, 255, 382

Hunsberger, S. D., Charlton, J. C., \& Zaritsky, D. 1998, ApJ, 505, 536

Klypin, A., Kravtsov, A. V., Valenzuela, O., \& Prada, F. 1999, ApJ, 522, 82

Kodaira, K., Sekiguchi, M., Sugai, H., \& Doi, M. 1991, PASJ, 43, 169

Koo, D. C., \& Kron, R. G. 1992, ARA\&A, 30, 613

Krusch, E., Bomans, D. J., Dettmar, R.-J., et al. The dwarf galaxy population in Hickson Compact Groups, submitted

Madgwick, D. S., Lahav, O., Baldry, I. K., et al. 2002, MNRAS, 333, 133

Mateo, M. L. 1998, ARA\&A, 36, 435

Mendes de Oliveira, C., \& Hickson, P. 1994, ApJ, 427, 684

Miles, T. A., Raychaudhury, S., Forbes, D. A., et al. 2004, MNRAS, 474, 3

Mobasher, B., et al. 2003, ApJ, 587, 605

Moore, B., Ghigna, S., Governato, F., et al. 1999, ApJ, 524, L19

Ribeiro, A. L. B., de Carvalho, R. R., \& Zepf, S. E. 1994, MNRAS, 267, L13

Sandage, A., Binggeli, B., \& Tammann, G. A. 1985, AJ, 90, 1759

Schechter, P. 1976, ApJ, 203, 297

Secker, J., \& Harris, W. E. 1997, PASP, 109, 1364

Trentham, N., \& Hodgkin, S. 2002, MNRAS, 333, 423

Trentham, N., \& Tully, R. B. 2002, MNRAS, 335, 712

Valdes, F. 1998, MSCRED Version 2.0

White, S. D. M., Briel, U. G., \& Henry, J. P. 1993, MNRAS, 261, L8

Zabludoff, A. I., \& Mulchaey, J. S. 1998, ApJ, 496, 39

Zabludoff, A. I., \& Mulchaey, J. S. 2000, ApJ, 539, 136

Zepf, S. E., \& Whitmore, B. C. 1991, ApJ, 383, 542 